\title{
Salient Region Detection Based on SLIC and Graph-based Segmentation Xiaofei Sun ${ }^{1, a}$, Wenwen Pan ${ }^{1, b}$, Xia Wang ${ }^{1, c}$, Xuhong Li ${ }^{1, d}$, Guan Wang ${ }^{1, e}$ \\ ${ }^{1}$ College of Information Science and Engineering, Zaozhuang University, Zaozhuang 277160, China \\ a 34740935@qq.com, b panwenwen@vip.qq.com, ' Wangxia1026@163.com \\ d87002898@qq.com, e289295022@qq.com
}

Keywords: saliency map; graph-based image segmentation; SLIC, color sparse histogram.

\begin{abstract}
At present, the most recent saliency detection algorithms are still not satisfactory. A saliency detection algorithm based on SLIC and graph-based segmentation is proposed. Firstly, graph-based segmentation is used to obtain larger image partitions, and the partitions with good contours are acquired. Then the relatively detailed partitions are obtained using SLIC image segmentation. Sparse color histogram is applied to these two methods. By using the color and spatial distance information, the saliency of each partition is calculated. Contours of salient objects from saliency maps generated using Graph-Based Segmentation are acquired and the gray scale values of the two kinds of saliency maps in the area surrounded by contours are merged. Experimental results show that compared with other detection methods, the proposed algorithm can effectively detect the salient object in the image.
\end{abstract}

\section{Introduction}

Image saliency detection is one of the challenging problems in computer vision. The purpose is to obtain a high quality of the saliency map. The saliency map shows the significant degree of different regions in the image. Saliency detection is used in many areas of computer vision, such as object detection, image segmentation, image retrieval, image compression, and so on[1-2].

In 1998, Itti of the University of California extracted the features of color, brightness, orientation and so on. The final saliency map is obtained by fusing these features[3]. Harel Jonathan proposed algorithm based on graph theory in 2006, the algorithm referred to as GBVS[4]. The algorithm structure is based on the Itti method, but the method based on graph is used in normalization. Cheng Mingming presents Global contrast based salient region detection in 2011. He uses a graph based image segmentation, combined with sparse color histogram to obtain a significant figure of high quality [5]. Zhang Qing proposed super pixel and histogram threshold based salient region detection algorithm in 2015 [6]. Wang Hailuo proposed super pixel fusion based salient region detection algorithm in 2015 [7]. These methods are generally used to compare the difference between the image unit and its adjacent image units in the gradation, color, direction or texture $[1,2,3]$. It is possible to compare the image unit with all the image units, and finally get a significant figure $[4,5$, $6,7]$.

Some of the above methods can be used to detect the edge of image, but can not effectively highlight the whole saliency of the target[1,2,3], and some methods do not have a good way to capture the target texture and edge details[6,7]. In view of this situation, a saliency detection method based on super pixel segmentation and sparse color histogram is proposed. This method retains the target texture and edge information by image segmentation based on graph, and then calculates the sparse color histogram by the SLIC super pixel. It uses a global comparison to ensure the integrity of the significant objectives, and to obtain a better effect of the significant figure.

\section{Graph-Based Image Segmentation and SLIC super pixel segmentation}

It is a classic image segmentation algorithm based on the greedy clustering algorithm. It is simple, fast and accurate. This algorithm can obtain the edge curve and texture of the object very well. The segmented regions are of the appropriate size. It does not produce too much of the "over segmentation" area. As shown in figure 1, the original image is segmented into partitions with 
similar color and texture features. Their outer contour, shape, and background are clearly divided.

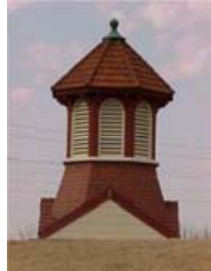

(a) original image

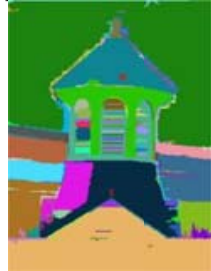

(b)result

Fig. 1 image segmentation using Graph-Based Segmentation algorithm

In this paper, we use Graph-Based Segmentation to make a preliminary segmentation of the original image. Because salient objects are generally close to the center of the picture, the probability of side partition color is set to the average probability of the central region color, when calculating the color histogram. This can highlight the saliency objects.

SLIC is a simple linear iterative clustering algorithm. The color image is transformed into CIELAB color space and the 5 dimensional feature vector in XY coordinates, then the distance metric is created for the 5 dimensional feature vector, finally the local clustering is performed on the image pixels. As shown in figure 2, SLIC algorithm can generate compact, approximate uniform super pixels, and its segmentation effect is more in line with people's expectations.

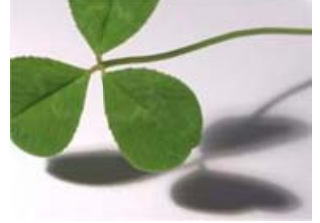

(a) original image

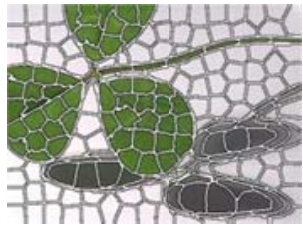

(b)result

Fig. 2 image segmentation using SLIC super pixel algorithm

First, image is segmented using the SLIC super pixel algorithm. Next, sparse color histograms in each super pixel are constructed. Then, color and position difference between super pixels is calculated. Finally, the saliency map is calculated.

\section{Saliency detection algorithm based on super pixel segmentation and sparse color histogram}

The existing research results of biological vision believe that the human visual system is very sensitive to the contrast of visual signals. The design of this algorithm is based on the following three aspects: (1) The contrast between the two partitions in the image depends on the color and the area. (2) The closer the distance between the partitions, the greater the impact they have on each other's saliency. (3) A saliency object generally close to the center of the image.

The main flow of the saliency feature extraction algorithm is shown in Figure 3.

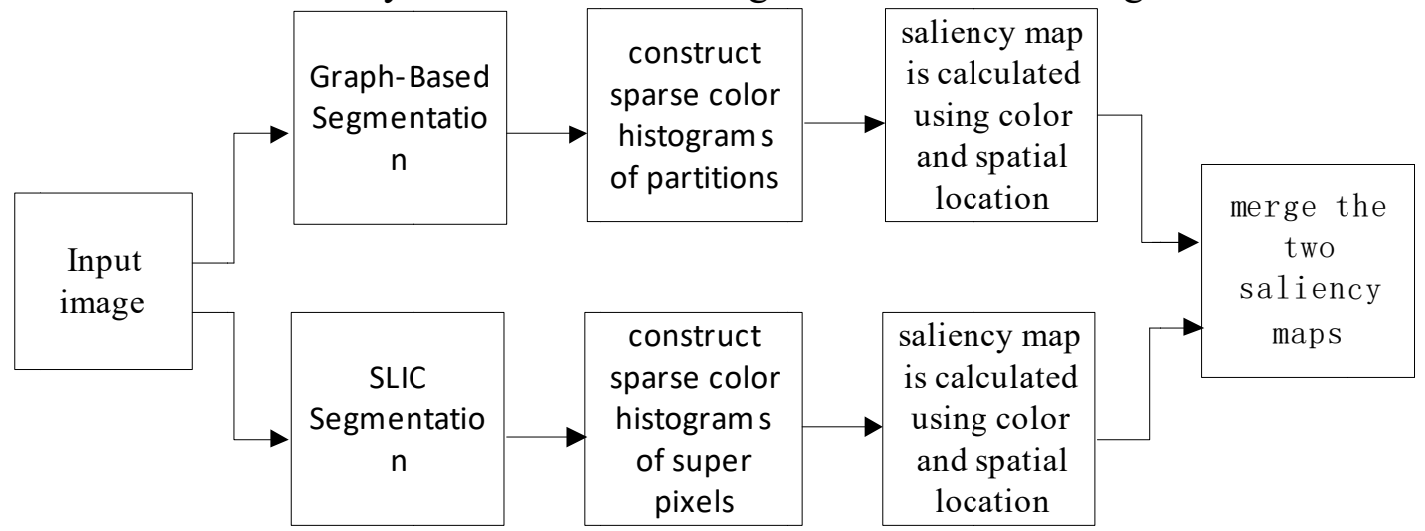

Fig. 3 Flow chart of the saliency feature extraction algorithm

The specific steps of the algorithm are as follows:

(1) The image is segmented using SLIC super pixel algorithm.

(2) Sparse color histograms of super pixels are constructed. The color number of each channel is reduced to 12 , so as to simplify the calculation and to keep the image contrast information. 
(3) Each color histogram is converted to Lab space, then color and distance differences between pixels are calculated.

$$
D_{p}\left(p_{m}, p_{n}\right)=\sum_{i=1}^{N_{1}} \sum_{j=1}^{N_{2}} f\left(c_{m, i}\right) f\left(c_{n, j}\right) D\left(c_{m, i}, c_{n, j}\right)
$$

$D\left(c_{m, i}, c_{n, j}\right)$ is the Euclidean distance between color $i$ in super pixel $p_{m}$ and color $j$ in super pixel $p_{n}$. N1 represents color number of super pixel $p_{m}$, and N2 represents color number of super pixel $p_{n}$.

It is generally believed that the impact of the near partition is greater on each other's saliency. The space distance between $p_{m}$ and $p_{n}$ pixels is calculated by the formula 2 .

$$
D_{s}\left(p_{m}, p_{n}\right)=\left|x_{m}-x_{n}\right|+\left|y_{m}-y_{n}\right|
$$

$D_{s}\left(p_{m}, p_{n}\right)$ is the distance between region $p_{m}$ and region $p_{m} \cdot x_{m}$ represents the $\mathrm{X}$ coordinate value of region $p_{m}$ centroid, and $y_{m}$ is the Y coordinate value of region $p_{m}$.

$$
S\left(p_{m}\right)=\sum_{i \neq m} \frac{w\left(p_{i}\right) D_{m}\left(p_{m}, p_{i}\right)}{\delta D_{s}\left(p_{m}, p_{i}\right)}
$$

$W\left(p_{i}\right)$ is the number of super pixel $p_{i}$. If the value is greater, the impact on the super pixel is greater. $\delta$ is an adjustment parameters according to the experience.

(4) The original image is initially segmented using Graph-Based Segmentation to obtain image partitions larger than super pixels.

(5) By repeating step (2)-(3), the saliency maps are generated using SLIC algorithm.

(6) Get contour of salient objects from saliency maps generated using Graph-Based Segmentation algorithm.

(7) Merge the gray scale values of the two kinds of saliency maps in the contour.

$$
\mathrm{g}(\mathrm{i})=\alpha g_{\text {graph }}(\mathrm{i})+\beta g_{\text {slic }}(\mathrm{i})
$$

$\mathrm{g}(\mathrm{i})$ is the final gray value of the pixel $i . g_{\text {graph }}(i)$ is the gray value of the pixel $i$ in saliency map generated using Graph-Based Segmentation. $g_{\text {slic }}(i)$ is the gray value of the pixel $i$ in saliency map generated using SLIC. $\alpha$ and $\beta$ are adjustment parameters based on the experience.

Saliency maps obtained using three kinds of algorithm are shown in figure 4 . From the figure we can see that salient region detection based on graph-based segmentation is good at detecting contour, but it detects texture and detail worse. Salient region detection based on SLIC is good at detecting texture and detail, but it detects contour worse. Merging algorithm proposed in this paper has best performance. 


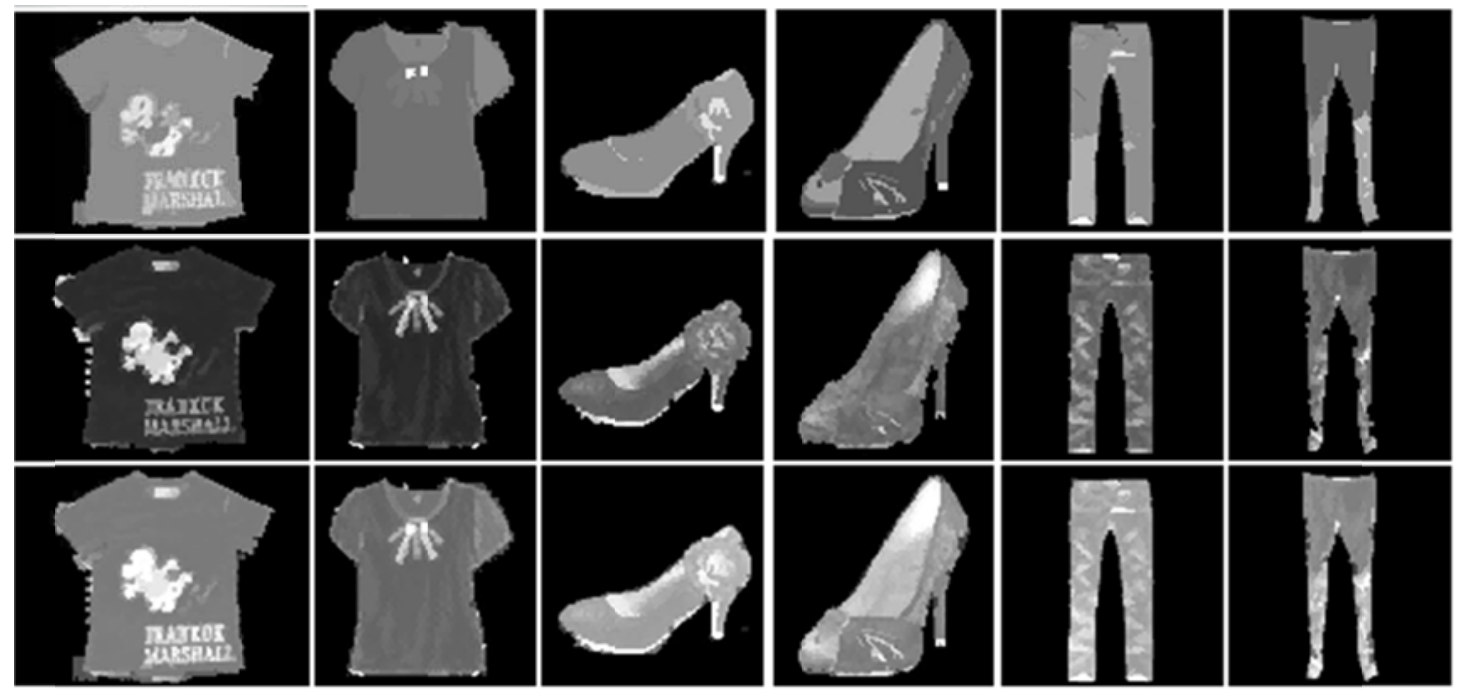

The pictures in the first row are generated by Graph-Based Segmentation. The pictures in the second row are generated by SLIC super pixel algorithm. The pictures in the third row are generated by the merging algorithm proposed in this paper.

Fig. 4 Comparison of three kinds of saliency map

\section{Conclusion}

In practice, salient region detection based on graph-based segmentation is good at detecting contour, but it leads to loss of texture and detail. Salient region detection based on SLIC is good at detecting texture and detail, but it usually leads to loss of contour. So we get contour of salient objects from saliency maps generated using Graph-Based Segmentation algorithm and merge the gray scale values of the two kinds of saliency maps in the contour. Sparse color histograms of super pixels are constructed and the color number of each channel is reduced in the process of calculating the saliency map. Final saliency map overcomes the shortcomings of the two algorithms, and retains the advantages of both, and can be used for image retrieval and so on. The algorithm can be widely used in Electronic Business, costume designing and artistic designing.

\section{Acknowledgments}

This work was financially supported by Zaozhuang university scientific research fund youth proj ect (grant No. 2015QN12).

\section{References}

[1] FRINTROP S, CHRISTENSEN H I. Computational visual attention systems and their cognitive foundations: A Survey [J]. ACM Transactions on Applied Perception, 2010,7(1).

[2] TREISMAN A M, GELADE G. A feature-integration theory of attention [J]. Cognitive Psychology, 1980, 12 (1).

[3] ITTI L, KOCH C, NIEBUR E. A Model of Saliency-based visual attention for rapid scene analysis [J]. IEEE Transactions on Pattern Analysis and Machine Intelligence, 1998, 20(11).

[4] HAREL J, KOCH C, PERONA P. Graph-based visual saliency[C]/Proceedings of the 2006 Conference Advances in Neural Information Processing Systems. Vancouver: MIT Press, 2006.

[5] Ming-Ming Cheng, Guo-Xin Zhang,et al.Global Contrast based Salient Region Detection. IEEE International Conference on Computer Vision and Pattern Recognition[C], 2011.409-416.

[6] WANG Hailuo, Wang Bo, ZHOU Zhiqiang, LI Sun, ZONG Hua. Superpixel-Fusion Based 
Salient Region Detection. Transactions of Beijing Institute of Technology[J]. 2015,8(35):836-841.

[7] ZHANG Qing, LIN Jiajun. Superpixel and histogram threshold based salient region detection. Computer Engineering and Applications[J]. 2015, 51(20):22-27.

[8] Deng Chengqiang, Feng Gang. A multi feature integrated image retrieval based on content. Computer application[J], 2003, 23(7):100-102.

[9] Xiaofei Sun, Wenwen Pan, Xia Wang, Wei Yuan. Image retrieval based on saliency detection in the application of the guide system. International Conference on Information Sciences[C], 2015.

[10] Wu Jie, Qing Zhengding. Progress in research on fusion underlying content features in image retrieval [J]. Chinese Journal of image and graphics, 2008, 13(2):189-197.

[11] $\mathrm{Hu} \mathrm{M}$. Visual pattern recognition by moment invariants [J].IRE Transactions on Information Theory, 1962,(8) :179-187. [11]Huang Yiping,Huang Xinsheng,Li Xiaolei. A new invariant of gray variation [J]. Journal of Dalian Maritime University, 2009, 34(4):23-27..

[12] Mingdru F, Tuytelaars T, Gool L V, et al. Moment invariants for recognition under changing viewpoint and illumination[J].Computer Vision and Image Understanding,2004,94(1):3-27.

[13] Lower D G. Distinctive image features from scale-invariant key points [J].International Journal of Computer Vision 2004, 60(2):91-110.

[14] Xiaofei Sun, Wenwen Pan, Xia Wang. Image retrieval with hand drawn draft based on saliency detection. ICISMME[C], 2015.

[15] Meng Lu. Color image salient region extraction based on visual attention mechanism [J]. Computer Application Research, 2013, 30(10):3159-3161.

[16] Yang Jun, Lin Tusheng, Xiao Yingwang. Salient object detection based on the global color contrast [J]. Computer Application Research, 2014.31(1):292-298. 